\title{
Multidimensional NQR Spectroscopy - A New Tool in Studies of Molecular Dynamics
}

\begin{abstract}
M. MAĆKOWIAK*
Institute of Molecular Physics, Polish Academy of Sciences

Smoluchowskiego 17, 60-179 Poznań, Poland

Two-dimensional exchange ${ }^{35} \mathrm{Cl}$ nuclear quadrupole resonance spectroscopy for studies of the $\mathrm{CCl}_{3}$-group reorientation processes in hexachloroethane and chloral hydrate has been applied. Experimental results were interpreted on the basis of the $2 \mathrm{D}$ exchange nuclear quadrupole resonance theory, which takes into account the off-resonance irradiation. It has been demonstrated that $2 \mathrm{D}$ nuclear quadrupole resonance exchange spectroscopy is appropriate for quantitative studies of exchange processes in molecular crystals containing quadrupolar nuclei. The method is of particular value for the detection of exchange networks in systems with many sites. Thus, detailed information on the exchange pathways within a network of structural isomers can be deduced and a proper assignment of the nuclear quadrupole resonance lines can be made. Temperature dependence of the exchange rate was studied. The mixing dynamics by exchange and the expected cross-peak intensities have been derived.
\end{abstract}

PACS numbers: 76.60.Gv, 82.56.Fk, 82.20.Pm

\section{Introduction}

Pure nuclear quadrupole resonance (NQR) spectroscopy is sensitive to structural, as well as dynamic, properties of the investigated system. In particular, NQR provides information on the quadrupolar interaction energy of the nuclear charge distribution with the electric field gradient (EFG) evaluated at the nuclear site. Molecular dynamics can be studied by pure NQR methods based on the measurement of spin-lattice relaxation times, lineshape parameters, and the temperature dependence of the resonance frequency. However, the amount of in-

*e-mail: mackow@ifmpan.poznan.pl 
formation provided by conventional one-dimensional (1D) NQR spectroscopy is very often limited. The $1 \mathrm{D}$ NQR method is unable to assign the specific lines of the multiline NQR spectrum to the particular reorienting molecular groups and to provide information on exchange-pathways.

Important improvements were achieved by introducing the two-dimensional (2D) techniques that offer fundamental advantages. The basic principle of $2 \mathrm{D}$ exchange NQR is the measurement of the NQR frequency of one and the same molecular segment at two different times and the detection of slow dynamics through a change in NQR frequency. 2D NMR exchange spectroscopy was first suggested by Jeener et al. [1]. Progress in multidimensional NQR has been hampered by experimental and conceptual difficulties. In contrast to NMR, some characteristic problems arise with a $2 \mathrm{D}$ exchange NQR experiment. The quantization axis is defined by the local direction of the EFG tensor. The direction of quantization changes jumpwise during the exchange process. With powder geometry, corresponding averages must be considered. Moreover, the NQR spectrum is usually spread over several hundreds $\mathrm{kHz}$. Thus the NQR lines are excited in off-resonance conditions. To our knowledge, only two attempts to use the pulse sequence of Jeener for NQR exchange studies exist in the literature [2, 3]. To explain many unsuccessful attempts we developed the theory, in which the mixing dynamics by exchange and the expected cross-peak intensities as a function of the frequency offset have been derived [4]. The application of this theory has allowed us to obtain 2D exchange NQR spectra in hexachloroethane and chloral hydrate, which is a subject of the present paper. The various features and practical applications of the proposed technique are demonstrated with experimental ${ }^{35} \mathrm{Cl} 2 \mathrm{D}$ exchange NQR spectra. The analysis of $2 \mathrm{D}$ spectra presented here allows one to assign the particular molecular groups and to provide information on molecular dynamics.

\section{Theory and method}

The basic scheme of $2 \mathrm{D}$ exchange spectroscopy involves four successive time periods: preparation, evolution, mixing, and detection. The transient response theory of a quadrupolar spin system to the three RF pulses (based on the solution of the time-dependent Schrödinger equation) has been given in [4], where it was shown that the time evolution of the signal created by pure NQR multipulse sequences is rather complicated. The basic pulse scheme of $2 \mathrm{D}$ exchange spectroscopy is given in Fig. 1. A schematic representation of the response of a system of quadrupolar spins to the three-pulse sequence is denoted by numbers, which correspond to the different subsequent signals. However, the most relevant term describing exchange interactions is the stimulated echo signal (SE) (denoted by 7 in Fig. 1). This is consequently the only term that gives rise to cross- and diagonal-peaks. We consider a system with two inequivalent sites $\mathrm{A}$ and $\mathrm{B}$ of the quadrupolar nuclei in a crystalline environment, with the NQR frequencies $\omega_{0}$ and $\omega_{0}^{\prime}$, respectively, among which the exchange takes place. For a nuclear spin $I=3 / 2$ 
the intensity of diagonal (AA) and cross (AB) peaks of the exchange spectrum is given by [4]

$$
\begin{aligned}
& G_{\mathrm{SE}}^{\mathrm{ex}}\left(t_{1}, t_{2}\right)=\frac{\alpha}{6 \rho^{2}} R^{2}(\theta, \varphi) \exp \left(-\frac{k}{2}\left(\tau_{\mathrm{m}}-t_{w}^{\prime}\right)\right)\left(P_{(\mathrm{AA})}+P_{(\mathrm{AB})}\right), \\
& P_{(\mathrm{AA})}=\frac{\sin \xi t_{w} \sin \xi t_{w}^{\prime} \sin \xi t_{w}^{\prime \prime}}{\xi^{3}}\left(K_{1}^{2}+K_{2} K_{2}^{*}\right) \cosh \left(\frac{k r}{2}\left(\tau_{\mathrm{m}}-t_{w}^{\prime}\right)\right) \\
& \times\left\{\frac{\Delta \omega}{2 \xi}\left[\sin \xi t_{w}^{\prime \prime}\left(\frac{\Delta \omega^{2}}{4 \xi^{2}} \sin \xi t_{w} \sin \xi t_{w}^{\prime}-\cos \xi t_{w} \cos \xi t_{w}^{\prime}\right)-\cos \xi t_{w}^{\prime \prime} \sin \xi\left(t_{w}+t_{w}^{\prime}\right)\right]\right. \\
& \times \cos \left(\omega_{0}\left(t_{2}-t_{1}\right)+\Delta \omega\left(t_{w}+t_{w}^{\prime \prime}\right)+\varphi_{1}+\varphi_{3}-\varphi_{2}\right) \\
& +\left[\cos \xi t_{w}^{\prime \prime}\left(-\frac{\Delta \omega^{2}}{4 \xi^{2}} \sin \xi t_{w} \sin \xi t_{w}^{\prime}+\cos \xi t_{w} \cos \xi t_{w}^{\prime}\right)-\frac{\Delta \omega^{2}}{4 \xi^{2}} \sin \xi t_{w}^{\prime \prime} \sin \xi\left(t_{w}+t_{w}^{\prime}\right)\right] \\
& \left.\times \sin \left(\omega_{0}\left(t_{2}-t_{1}\right)+\Delta \omega\left(t_{w}+t_{w}^{\prime \prime}\right)+\varphi_{1}+\varphi_{3}-\varphi_{2}\right)\right\}, \\
& P_{(\mathrm{AB})}=\frac{\sin \xi^{\prime} t_{w} \sin \xi^{\prime} t_{w}^{\prime} \sin \xi t_{w}^{\prime \prime}}{\xi^{\prime 2} \xi}\left(K_{1}^{\prime 2}+K_{2}^{\prime} K_{2}^{{ }^{*}}\right) \sinh \left(\frac{k r}{2}\left(\tau_{\mathrm{m}}-t_{w}^{\prime}\right)\right) \\
& \times\left\{\left[\frac{\Delta \omega}{2 \xi} \sin \xi t_{w}^{\prime \prime}\left(\frac{\Delta \omega^{\prime 2}}{4 \xi^{\prime 2}} \sin \xi^{\prime} t_{w} \sin \xi^{\prime} t_{w}^{\prime}-\cos \xi^{\prime} t_{w} \cos \xi^{\prime} t_{w}^{\prime}\right)\right.\right. \\
& \left.-\frac{\Delta \omega^{\prime}}{2 \xi^{\prime}} \cos \xi t_{w}^{\prime \prime} \sin \xi^{\prime}\left(t_{w}+t_{w}^{\prime}\right)\right] \cos \left(\omega_{0} t_{2}-\omega_{0}^{\prime} t_{1}+\Delta \omega^{\prime}\left(t_{w}+t_{w}^{\prime \prime}\right)+\varphi_{1}+\varphi_{3}-\varphi_{2}\right) \\
& +\left[\cos \xi t_{w}^{\prime \prime}\left(-\frac{\Delta \omega^{\prime 2}}{4 \xi^{\prime 2}} \sin \xi^{\prime} t_{w} \sin \xi^{\prime} t_{w}^{\prime}+\cos \xi^{\prime} t_{w} \cos \xi^{\prime} t_{w}^{\prime}\right)\right. \\
& \left.-\frac{\Delta \omega \Delta \omega^{\prime}}{4 \xi \xi^{\prime}} \sin \xi t_{w}^{\prime \prime} \sin \xi^{\prime}\left(t_{w}+t_{w}^{\prime}\right)\right] \\
& \left.\times \sin \left(\omega_{0} t_{2}-\omega_{0}^{\prime} t_{1}+\Delta \omega^{\prime}\left(t_{w}+t_{w}^{\prime \prime}\right)+\varphi_{1}+\varphi_{3}-\varphi_{2}\right)\right\},
\end{aligned}
$$

where $\alpha=\frac{\gamma B_{1}}{4}, \rho=\sqrt{1+\frac{\eta^{2}}{3}}, R(\theta, \varphi)=\sqrt{4 \eta^{2} \cos ^{2} \theta+\left(9+\eta^{2}+6 \eta \cos 2 \varphi\right) \sin ^{2} \theta}$, $k$ is the average exchange rate, and $r$ is a reduction factor taking into account the losses of the spin-polarization projection during the reorientational jumps. The spectrometer frequency $\omega$ may be different from the resonance frequency $\omega_{0}$ by $\Delta \omega=\omega-\omega_{0} ; \phi_{1}, \phi_{2}$, and $\phi_{3}$ are the phases of the pulses, respectively. Here $K_{1}=\frac{2 \alpha \eta}{\sqrt{3} \rho} \cos \theta$ and $K_{2}=-\frac{\alpha \sin \theta}{\sqrt{3} \rho}\left(3 \mathrm{e}^{-\mathrm{i} \varphi}+\eta \mathrm{e}^{\mathrm{i} \varphi}\right)$. The variables $\xi$ are defined as $\xi=\frac{1}{2} \sqrt{4 m^{2}+\Delta \omega^{2}}$, where $m=\frac{\alpha R(\theta, \varphi)}{\sqrt{3} \rho}$. 


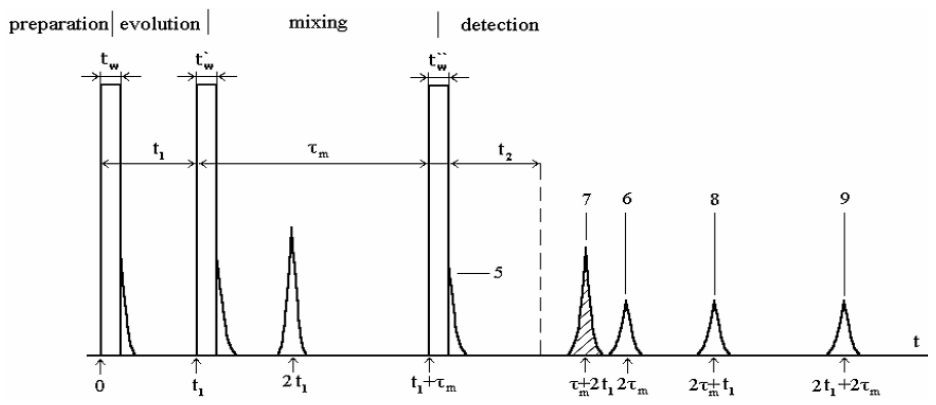

Fig. 1. Basic pulse scheme of $2 \mathrm{D}$ exchange spectroscopy. Schematic representation of the response of a system of quadrupolar spins to the three-pulse sequence. The subsequent NQR signals after the third pulse are denoted by numbers.

The part $P_{(\mathrm{AA})}$ expression in (1) describes the diagonal-peak with coordinates $\left[\omega_{0}, \omega_{0}\right]$ whereas the $P_{(\mathrm{AB})}$ refers to the cross-peak with coordinates $\left[\omega_{0}, \omega_{0}^{\prime}\right]$ in the $2 \mathrm{D}$ NQR spectrum. The signal intensities and phases of the diagonal and cross-peaks in powders were analysed as a function of the pulse length and the relative frequency offset of the spectrometer frequency $\Delta \omega_{0} / \omega_{r}$. The analysis was performed for various parameters $\Delta=\left(\Delta \omega_{\mathrm{B}}-\Delta \omega_{\mathrm{A}}\right) / \omega_{\mathrm{r}}$, where $\omega_{r}=\gamma B_{i}$. Figure 2 shows the ratio of the cross-peaks ( $\mathrm{AB}$, curve 1 ; BA, curve 2 ) and diagonal-peak (AA) intensities as a function of the relative frequency offset. The consequence of the frequency offset is that the intensity ratio can change drastically from almost comparable to near zero values. It is interesting to note that the diagonal- and cross-peaks intensities are very weakly dependent on the EFG-tensor asymmetry parameter as well as the angles $\alpha$ and $\delta$ that determine the positions of exchange atoms in a molecule.

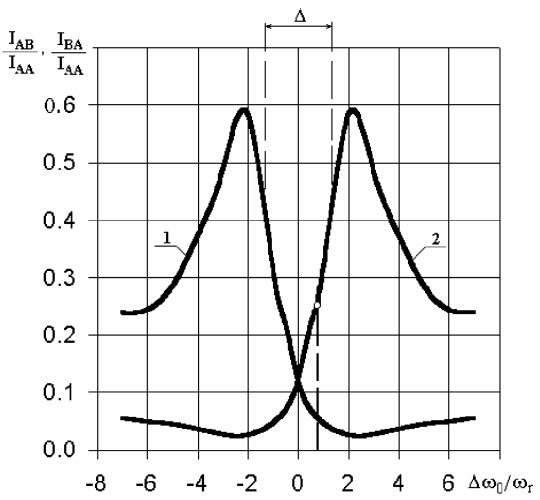

Fig. 2. Ratio of the cross-peaks (AB - curve 1, BA - curve 2) and diagonal peak (AA) intensity as a function of the relative frequency offset. 


\section{Experimental}

2D exchange ${ }^{35} \mathrm{Cl} \mathrm{NQR}$ experiments were carried out with polycrystalline hexachloroethane, $\mathrm{C}_{2} \mathrm{Cl}_{6}$, and chloral hydrate, $\mathrm{CCl}_{3} \mathrm{CH}(\mathrm{OH})_{2}$. The samples were purchased from Aldrich Chemical Co. The purity was specified as "pro analysi" (99\%). The spectra have been recorded with a digital NMR Bruker spectrometer DSX 200 equipped with a special NQR probehead. The temperature was measured and controlled using the Bruker variable temperature unit BVT 3000. The standard NMR software UXNMR was used. For the 2D exchange experiments the noesytp pulse sequence (equivalent to the $2 \mathrm{D}$ homonuclear correlation via dipolar coupling in NMR spectroscopy) was used. The typical length of an RF pulse for the optimum ${ }^{35} \mathrm{Cl}$ signal excitation was $3.5 \mu \mathrm{s}$. As shown in our earlier paper [4], for such a short pulse the off-resonance effects preventing detection of the cross-peaks were compensated.

\section{Results and discussion}

${ }^{35} \mathrm{Cl}$ Fourier-transformed NQR spectrum of hexachloroethane recorded at $233 \mathrm{~K}$ is shown in Fig. 3. The pure ${ }^{35} \mathrm{Cl} \mathrm{NQR}$ frequencies of $\mathrm{C}_{2} \mathrm{Cl}_{6}$ are $\nu_{\mathrm{A}}=40.117 \mathrm{MHz}, \nu_{\mathrm{B}}=\nu_{\mathrm{C}}=40.206 \mathrm{MHz}, \nu_{\mathrm{D}}=40.247 \mathrm{MHz}$, and $\nu_{\mathrm{E}}=\nu_{\mathrm{F}}=$ $40.260 \mathrm{MHz}$ (at $T=233 \mathrm{~K}$ ). With a frequency separation of $143 \mathrm{kHz}$, all lines can be excited simultaneously within the bandwidth of conventional RF pulses, but under off-resonance conditions. The chlorine nuclei B and C (and similarly E and $\mathrm{F}$ ) are located in equivalent surroundings and are subject to the same resonance frequency. The specific NQR lines in the one-dimensional spectrum cannot be assigned to any particular $\mathrm{CCl}_{3}$-group. This problem can be solved only by $2 \mathrm{D}$ exchange spectroscopy, which provides a means to visualise the exchange pathway pattern of a given compound and to distinguish between the possible models of exchange. In $\mathrm{C}_{2} \mathrm{Cl}_{6}$ two models of exchange have to be considered. In the first case one can assume that rotational jumps interchange the site of the chlorine nucleus A with one of the two sites of the nuclei $\mathrm{B}$ or $\mathrm{C}$ (it means that the nuclei $\mathrm{A}, \mathrm{B}$, and $\mathrm{C}$ belong to one $\mathrm{CCl}_{3}$-group). The second model considered involves the possible interchange of the site of $\mathrm{A}$ with that of $\mathrm{E}$ or $\mathrm{F}$ that corresponds to another assignment of the $\mathrm{CCl}_{3}$-group. Other combinations are less probable but in principle cannot be excluded.

Figure 4 shows the ${ }^{35} \mathrm{Cl} 2 \mathrm{D}$ exchange NQR spectrum of hexachloroethane recorded at $T=233 \mathrm{~K}$. Cross- and diagonal-peaks are clearly visible. The mixing interval was $\tau_{\mathrm{m}}=2 \mathrm{~ms}$. The evolution period $t_{1}$ was incremented in 128 steps of $1 \mu \mathrm{s}$. In the $t_{2}$ interval 512 data points in steps of $0.5 \mu \mathrm{s}$ were taken. The number of accumulations was 6000 , the repetition time was $20 \mathrm{~ms}$, and the total time of the experiment was $6 \mathrm{~h}$. The two-cross sectional spectra positioned at the NQR frequencies $40.117 \mathrm{MHz}$ and $40.206 \mathrm{MHz}$ are shown in Fig. 4. The spectra demonstrate the large intensity ratio of diagonal-peaks (high) and crosspeaks (low). As shown in Fig. 4, only one pair of cross-peaks was detected. It has 


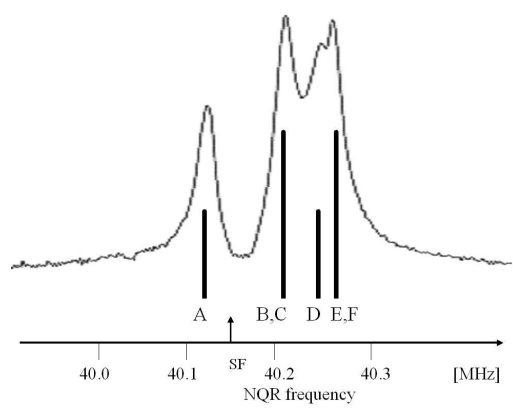

Fig. 3. ${ }^{35} \mathrm{Cl}$ NQR spectrum of hexachloroethane recorded at $233 \mathrm{~K}$. The corresponding stick-spectrum is also shown. The spectrometer frequency (SF) is $40.150 \mathrm{MHz}$.

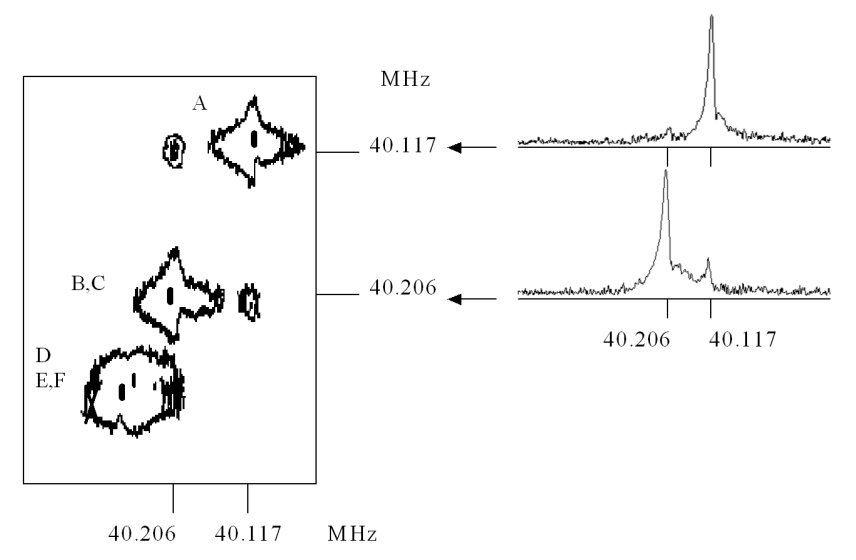

Fig. 4. 2D exchange NQR spectrum of hexachloroethane recorded with ${ }^{35} \mathrm{Cl}$ signals at $233 \mathrm{~K}$. The mixing time was $2 \mathrm{~ms}$. The transmitter frequency was $40.150 \mathrm{MHz}$. The two-cross sectional spectra positioned at the NQR frequencies $40.117 \mathrm{MHz}$ and $40.206 \mathrm{MHz}$ of one of the dimensions in the frequency domain illustrate the large intensity ratio of diagonal- (high) and cross-peaks (low).

unambiguously been shown that the first motional model is valid. It means that the atoms $\mathrm{A}, \mathrm{B}$, and $\mathrm{C}$ belong to the one $\mathrm{CCl}_{3}$-group, whereas atoms $\mathrm{D}, \mathrm{E}$, and $\mathrm{F}$ belong to another group. The expected cross-peaks between the lines D, E, and F are not visible because of low resolution.

The crystal structure of hexachloroethane $\left(\mathrm{C}_{2} \mathrm{Cl}_{6}\right)$ is rhombic, space group $D_{2 n}^{16}=P_{n m a}$, with four molecules per unit cell [5]. Figure 5 shows the molecular structure of hexachloroethane. Here pure tetrahedral symmetry for the $\mathrm{CCl}_{3}$-groups is assumed for simplicity. The chlorine nuclei are located in three corners of the tetrahedra. Thus the rotational jump of the $\mathrm{CCl}_{3}$-group, concluded from relaxation data [6], interchanges the position of chlorine nuclei. This reorientation is equivalent to the rotation along the $\mathrm{C}-\mathrm{C}$ bond axis by an angle 


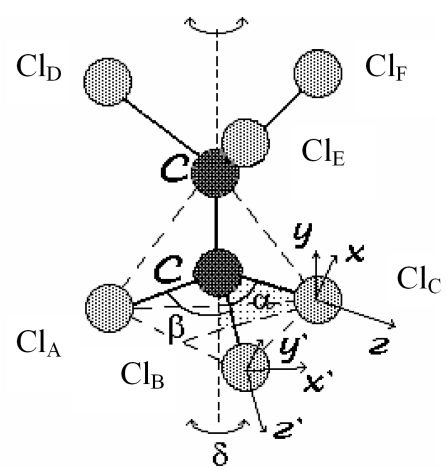

Fig. 5. Molecular structure of hexachloroethane.

$\delta= \pm 120^{\circ}(\alpha=\arcsin (4 / 3 \sqrt{2}), \beta=\arcsin (2 / \sqrt{6})$. The coordinate system connected with the EFG principal axes will be transformed by the rotation matrix. The consequence of the rapid reorientational jump is that only the projection of the old spin polarization on the new axis of quantization is relevant for the coherence pathway leading to the exchange cross-peak. Thus the $z z$-components of the EFG tensor, determining the cross-peak intensity in the two-dimensional exchange spectrum, are transformed as

$$
V_{z z}^{\prime}=r V_{z z}
$$

where the reduction factor $r$, taking into account these projection losses of the exchange peak intensity, is defined as

$$
\begin{aligned}
r= & \frac{1}{2}\left\{2\left(\cos ^{2} \alpha+\cos \delta \sin ^{2} \alpha\right)^{2}-(1-\cos \delta)^{2} \sin ^{2} \alpha \cos ^{2} \alpha\right. \\
& \left.-\sin ^{2} \delta \sin ^{2} \alpha+\eta\left(\sin ^{2} \alpha\right)\left[(1-\cos \delta)^{2} \cos ^{2} \alpha-\sin ^{2} \delta\right]\right\} .
\end{aligned}
$$

For a system with tetrahedral angles we can assume $\delta= \pm 180^{\circ}, \beta=2 \alpha=$ $120^{\circ}$, and Eq. (3) takes the following form:

$$
V_{z z}^{\prime}=r V_{z z}=\frac{1}{2}\left(3 \cos ^{2} \beta-1+\eta \sin ^{2} \beta\right) V_{z z}
$$

The value of the reduction factor $r$ for $\mathrm{C}_{2} \mathrm{Cl}_{6}$ is -0.333 .

In a series of further experiments, the mixing time was varied. The resulting cross- and diagonal-peak intensities as a function of the mixing time $\tau_{\mathrm{m}}$ are plotted in Fig. 6. As shown in $[4,7]$, the signal intensities of diagonal- and cross-peaks, obtained for a mixing time $\tau_{\mathrm{m}}$, are described by the mixing coefficients $\alpha_{\mathrm{AA}}$ and $\alpha_{\mathrm{AB}}$, respectively. For the exchanging $\mathrm{CCl}_{3}$-group, the mixing coefficient, which describes the intensity of diagonal peaks, is given by

$$
\alpha_{\mathrm{AA}}=\frac{2}{9} \exp \left(\frac{-6 R-4 k+k r}{6} \tau_{\mathrm{m}}\right)\left[\exp \left(\frac{k r}{2} \tau_{\mathrm{m}}\right)+2 \cosh \left(\frac{k r}{2} \tau_{\mathrm{m}}\right)\right] .
$$

For the cross-peaks the mixing coefficient is 


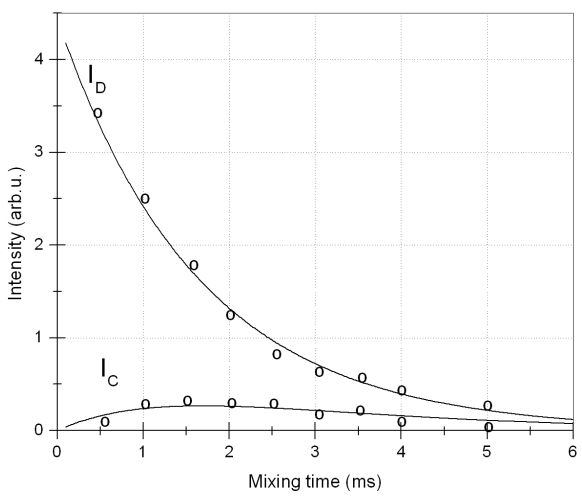

Fig. 6. Intensities $I_{\mathrm{D}}$ and $I_{\mathrm{C}}$ of diagonal- and cross-peaks, respectively, of hexachloroethane at $233 \mathrm{~K}$ as a function of the mixing time $\tau_{\mathrm{m}}$. The solid lines provide the theoretical dependence calculated for the following model parameters: $k=288 \mathrm{~s}^{-1}$, $R=385 \mathrm{~s}^{-1}, E_{\mathrm{a}}=75.8 \mathrm{~kJ} \mathrm{~mol}^{-1}, T=233 \mathrm{~K}, r=-0.333$, and $\tau_{0}=3.5 \times 10^{-20} \mathrm{~s}$.

$$
\alpha_{\mathrm{AB}}=\frac{4}{9} \exp \left(\frac{-6 R-4 k+k r}{6} \tau_{\mathrm{m}}\right) \sinh \left(\frac{k r}{2} \tau_{\mathrm{m}}\right),
$$

where $k$ is the average exchange rate, $R=1 / T_{1}$ is the relaxation rate, and $r$ is a reduction factor taking into account the losses of the spin-polarization projection during the reorientational jumps.

The exchange rate, $k$, can be expressed by the activation energy, $E_{\mathrm{a}}$, using the relation

$$
k=\tau_{0}^{-1} \exp \left(-\frac{E_{\mathrm{a}}}{R T}\right),
$$

where $\tau_{0}$ is the extrapolated correlation time.

According to Goldman's theory [8] for the reorienting $\mathrm{CCl}_{3}$-group, the spin-lattice relaxation time $T_{1}$ is related to the correlation time, $\tau_{\mathrm{c}}$, by a simple formula

$$
T_{1}=\frac{3 \tau_{\mathrm{c}}}{4}=\frac{3 \tau_{0}}{4} \exp \left(\frac{E_{\mathrm{a}}}{R T}\right)=\frac{3}{4 k} .
$$

Thus $R=4 k / 3$ and this value was used for fitting the experimental results by Eqs. $(5,6)$. The solid lines in Fig. 6 are theoretical dependence calculated for the following model parameters: $k=288 \mathrm{~s}^{-1}, R=385 \mathrm{~s}^{-1}, E_{\mathrm{a}}=75.8 \mathrm{~kJ} \mathrm{~mol}^{-1}$, $T=233 \mathrm{~K}, r=-0.333$, and $\tau_{0}=3.5 \times 10^{-20} \mathrm{~s}$. Very good agreement of experimental results with theoretical predictions confirms that the $\mathrm{CCl}_{3}$-group in chloral hydrate underlies a three-fold rotational symmetry. Using these parameters the simulated 2D exchange NQR spectrum (contour plot) was calculated. As shown in Fig. 7, the full agreement between the experimental and theoretical spectrum has been found. It confirms the motional model and dynamic parameters determined from the experiment. 


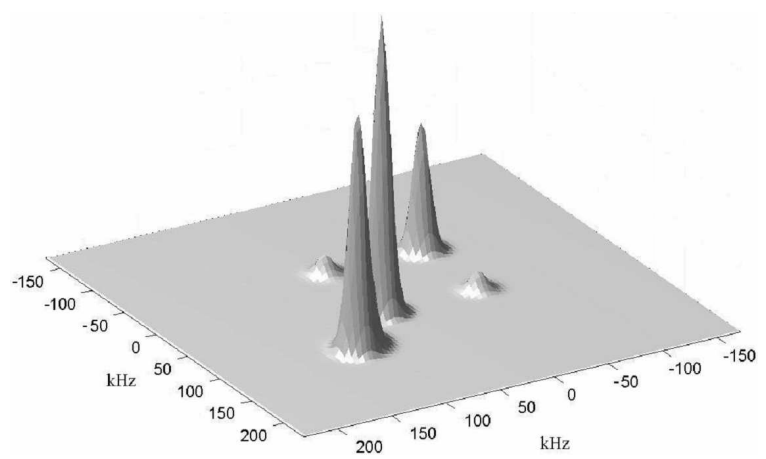

Fig. 7. Simulated $2 \mathrm{D}$ exchange NQR spectrum in the powder sample of $\mathrm{C}_{2} \mathrm{Cl}_{6}$.

In a second series of experiments, the temperature was varied. The measurements were performed in the temperature range of $215-235 \mathrm{~K}$. Below $215 \mathrm{~K}$ the cross-peaks are not visible, because the reorientation is hindered. Above $240 \mathrm{~K}$ the NQR spectrum disappears due to the fast rotation of the $\mathrm{CCl}_{3}$-group and shortening of the spin-lattice relaxation time ("fading-out phenomenon"). As follows from Eqs. $(5,6)$, the ratio of the mixing coefficients of the cross- and diagonal-peaks is given by

$$
\gamma=\frac{\alpha_{\mathrm{AB}}}{\alpha_{\mathrm{AA}}}=\frac{I_{\mathrm{C}}}{I_{\mathrm{D}}}=\frac{2 \sinh \left(k r \tau_{\mathrm{m}} / 2\right)}{\exp \left(-k r \tau_{\mathrm{m}} / 2\right)+2 \cosh \left(k r \tau_{\mathrm{m}} / 2\right)} .
$$

Thus

$$
x=k r \tau_{\mathrm{m}}=\ln \left(\frac{1+2 I_{\mathrm{C}} / I_{\mathrm{D}}}{1-I_{\mathrm{C}} / I_{\mathrm{D}}}\right) .
$$

Activation energy of the exchange process can be determined on this basis assuming an Arrhenius law (see Eq. (7)). From the temperature dependence of $\ln (x)$ presented in Fig. 8 one can obtain the value of activation energy in the temperature range of $215-235 \mathrm{~K}, E_{\mathrm{a}}=75.8 \mathrm{~kJ} \mathrm{~mol}^{-1}$. Such a large value of the reorientational potential barrier suggests the reorientations of the $\mathrm{C}_{2} \mathrm{Cl}_{6}$ molecule as a whole in the crystal lattice.

In chloral hydrate at $300 \mathrm{~K}$ the NQR frequencies were measured to be $\nu_{1}=37.476 \mathrm{MHz}, \nu_{2}=38.664 \mathrm{MHz}$, and $\nu_{3}=38.750 \mathrm{MHz}$, and the relaxation time $T_{1}$ is $3 \mathrm{~ms}$. With a frequency separation of $86 \mathrm{kHz}$, both $\nu_{2}$ and $\nu_{3}$ lines can be excited simultaneously within the bandwidth of conventional RF pulses. An exponential change of $T_{1}(T)$ function above $250 \mathrm{~K}$ was interpreted as arising from the hindered rotation of the $\mathrm{CCl}_{3}$-group $[9,10]$. Figure 9 shows the $2 \mathrm{D}$ exchange NQR magnitude spectrum of chloral hydrate recorded at $T=300 \mathrm{~K}$. Cross- and diagonal-peaks are clearly visible. The mixing interval was $\tau_{\mathrm{m}}=3 \mathrm{~ms}$. The evolution period $t_{1}$ was incremented in 256 steps. In the $t_{2}$ interval 64 data points were taken. The number of accumulations was 32000 at the repetition time $15 \mathrm{~ms}$ and the total experiment time $-12 \mathrm{~h}$. 


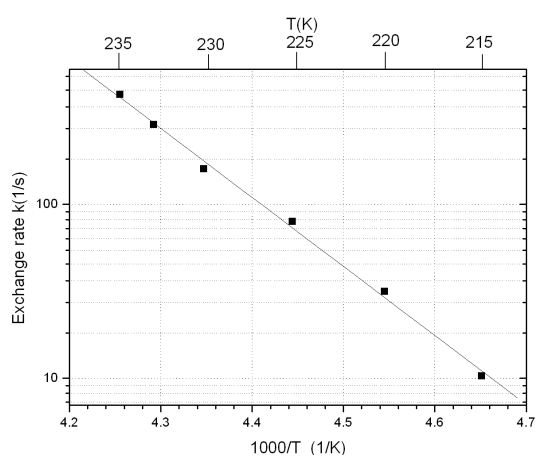

Fig. 8. Temperature dependence of exchange rates in hexachloroethane.

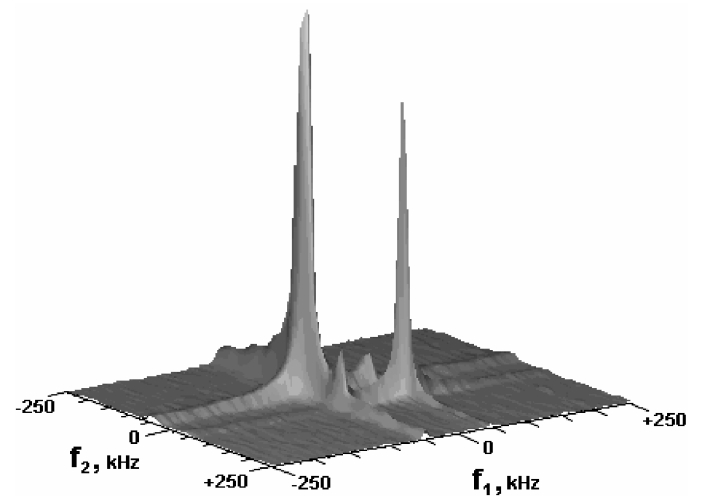

Fig. 9. 2D exchange NQR (magnitude) spectrum in chloral hydrate recorded with $35 \mathrm{Cl}$ signals at $T=300 \mathrm{~K}$.

The cross- and diagonal-peak intensities as a function of the mixing time $\tau_{\mathrm{m}}$ are plotted in Fig. 10. The solid lines in Fig. 10 provide the theoretical dependence calculated for the following model parameters: $k=250 \mathrm{~s}^{-1}, R=333 \mathrm{~s}^{-1}$, $E_{\mathrm{a}}=14.5 \mathrm{kcal} \mathrm{mol}^{-1}, T=300 \mathrm{~K}, r=-0.333$, and $\tau_{0}=1.09 \times 10^{-13} \mathrm{~s}$. The very good agreement between the experimental results and the theoretical predictions confirms that the motion of the $\mathrm{CCl}_{3}$-group in chloral hydrate is characterized by a three-fold rotational symmetry.

The temperature dependence of the diagonal- and cross-peaks intensities is shown in Fig. 11. The measurements were performed in the temperature range of 240-310 K. Below $240 \mathrm{~K}$ the cross-peaks are not visible, because the reorientation is hindered. Above $310 \mathrm{~K}$ the NQR spectrum disappears due to the fast rotation of the $\mathrm{CCl}_{3}$-group and shortening of the spin-lattice relaxation time ("fading-out phenomenon"). From the temperature dependence of $\ln (x)$ presented in Fig. 5 one can conclude that the activation energy is different in the temperature ranges of $240-290 \mathrm{~K}$ and of $290-310 \mathrm{~K}$. The obtained results are $E_{\mathrm{a}}=7.1 \mathrm{kcal} \mathrm{mol}^{-1}$ and $E_{\mathrm{a}}=14.5 \mathrm{kcal} \mathrm{mol}^{-1}$, respectively. This suggests that beside the hindered 


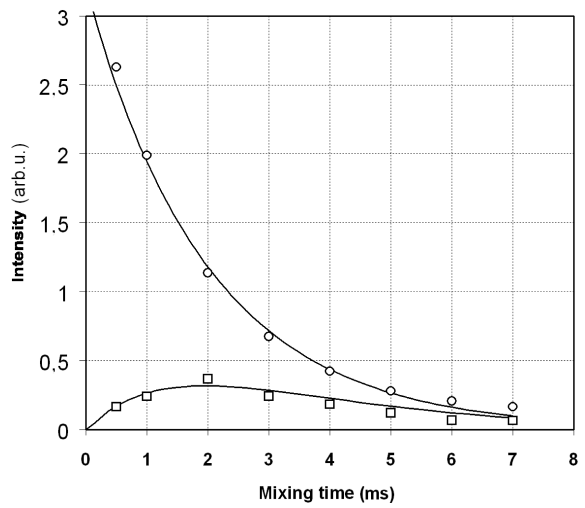

Fig. 10. Intensities of diagonal- $I_{\mathrm{D}}(\circ)$ and cross-peaks $5 I_{\mathrm{C}}(\square)$ of chloral hydrate at $300 \mathrm{~K}$ as a function of the mixing time $\tau_{\mathrm{m}}$. The solid lines are theoretical dependence calculated for the following model parameters: $k=250 \mathrm{~s}^{-1}, R=333 \mathrm{~s}^{-1}, E_{\mathrm{a}}=$ $14.5 \mathrm{kcal} \mathrm{mol}^{-1}, T=300 \mathrm{~K}, r=-0.333$, and $\tau_{0}=1.09 \times 10^{-13} \mathrm{~s}$.

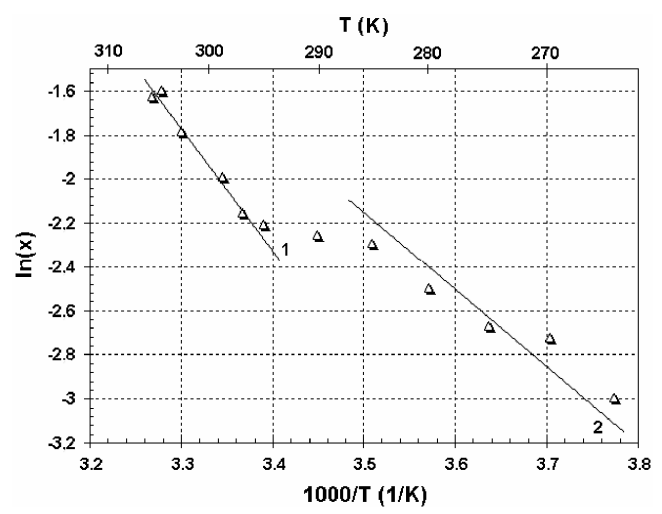

Fig. 11. Temperature dependence of exchange rates in chloral hydrate.

rotation of the $\mathrm{CCl}_{3}$ - group activated above $240 \mathrm{~K}$, there is another mechanism of relaxation that is activate above $290 \mathrm{~K}$.

The analysis of the known crystallographic structure of chloral hydrate leads us to the conclusion that this other mechanism is related to an order-disorder transition in which the hydrogen bonds are broken. It seems justified to assume that above $295 \mathrm{~K}$ the quadrupole relaxation is a result of the joint influence of correlated rotation of the chloral hydrate molecule as a whole unit and breaking of the hydrogen bonds.

\section{Conclusions}

With the present study, it has been demonstrated that 2D NQR exchange spectroscopy is appropriate for quantitative studies of exchange processes in molec- 
ular crystals containing quadrupole nuclei. The method is of particular value for the detection of exchange networks in systems with many sites. Thus, detailed information on the exchange pathways within a network of structural isomers can be deduced and a proper assignment of the NQR lines can be made. This piece of information is inaccessible by other techniques. The mixing dynamics by exchange and the expected cross-peak intensities have been derived. The very good agreement of the experimental results with theoretical predictions confirms the validity of the motion model. 2D exchange NQR spectroscopy in hexachloroethane and chloral hydrate has allowed us to measure the activation energy and other molecular dynamic parameters.

\section{Acknowledgments}

The author is highly indebted to Prof. B. Bluemich (Aachen) for permission to use a Bruker spectrometer DSX 200 for taking experimental data. The assistance of Prof. N. Sinyavsky (Kaliningrad) in theoretical calculations is very appreciated.

The work was supported by the Centre of Excellence for Magnetic and Molecular Materials for Future Electronics within the European Commission Contract No. G5MA-CT-2002-04049.

\section{References}

[1] J. Jeener, B.H. Maier, P. Bachmann, R.R. Ernst, J. Chem. Phys. 71, 4546 (1979).

[2] E. Rommel, P. Nickel, F. Rohmer, R. Kimmich, C. Gonzales, D. Pusiol, Z. Naturforsch. A 47, 382 (1992).

[3] N. Sinyavsky, M. Maćkowiak, B. Bluemich, Z. Naturforsch. A 57, 53 (2002).

[4] N. Sinyavsky, N. Velikite, M. Maćkowiak, Mol. Phys. 99, 1653 (2001).

[5] Y. Sasada, M. Atoji, J. Chem. Phys. 21, 145 (1953).

[6] Yu.N. Gachegov, A.D. Gordeev, G.B. Soifer, J. Mol. Struct. 83, 109 (1982).

[7] P. Nickel, R. Kimmich, J. Mol. Struct. 345, 253 (1995).

[8] M. Goldman, Adv. Magn. Reson. 14, 59 (1990).

[9] H. Chihara, N. Nakamura, Bull. Chem. Soc. Jpn. 45, 3530 (1972).

[10] J. Kasprzak, J. Pietrzak, A. Pietrzak, J. Mol. Struct. 192, 379 (1989). 